\title{
Meningeal tumor: A rare extrahepatic association in patients with polycystic liver disease enrolled for liver transplantation
}

\author{
ALEXANDRE BAKONYI NETO ${ }^{1}$, MARCO ANTONIO ZANINI ${ }^{2}$, AMANDA PINTER CAVALHEIRO DA SILVA ${ }^{1}$, \\ CAMILA WINCKLER ${ }^{1}$, RODRIGO MATTOS DOS SANTOS ${ }^{3}$ and MARCELO LOPES FURTADO ${ }^{1}$ \\ Departments of ${ }^{1}$ Surgery, Division of Gastrointestinal Transplant, ${ }^{2}$ Neurosurgery, and ${ }^{3}$ Urology, \\ Botucatu Faculty of Medicine, UNESP, Botucatu, SP, Brazil
}

Received August 5, 2011; Accepted November 14, 2011

DOI: $10.3892 / \mathrm{ol} .2012 .605$

\begin{abstract}
In the present study, we described a rare association of polycystic liver disease (PCLD) with intracranial meningiomas in patients included on a liver transplant list, focusing on the diagnosis, treatment and possible association with any genetic alterations. Two female patients, aged 39 and 49 years were included on a liver transplant list due to extensive PCLD, with symptoms related to an abdominal compartmental syndrome. Screening for extrahepatic manifestation revealed a right frontal meningioma in the first patient, and a parietal posterior calcified meningioma in the second patient, measuring 1 and $7 \times 3 \times 2 \mathrm{~cm}$ in diameter, respectively. Following tumor removal, the histological pattern was compatible with fibrous and transitional meningioma, respectively. Cytogenetic studies conducted following surgery did not reveal any changes in metaphase chromosomes. The postoperative follow-up for the two patients was uneventful, without complications, with the patients remaining on a liver transplant waiting list. We conclude that screening for extrahepatic manifestations of PCLD is mandatory, as certain lesions require treatment prior to liver transplantation. The lack of a genetic or familial association between these two cases show they are likely to have occurred by chance, rather than representing a previously unrecognized association between polycystic liver disease and cranial meningioma.
\end{abstract}

\section{Introduction}

Intracranial manifestations correlated with autosomal dominant polycystic kidney disease (ADPKD), including intraparenchymal hemorrhage and aneurysm formation, have been widely described (1), as has an increased incidence of intracranial arachnoid cysts $(1,2)$. Hepatic cysts are relatively common in patients with $\operatorname{ADPKD}(3,4)$, increasing with advancing age,

Correspondence to: Dr Alexandre Bakonyi Neto, Alameda Antonio Sartor 220, Parque das Cascatas, 18607-340 Botucatu, SP, Brazil

E-mail: a.bakonyi.neto@hotmail.com

Key words: meningioma, liver transplantation, polycystic liver disease female gender, pregnancy and degree of renal lesions, which affect the course of polycystic liver disease (PCLD) (5). The majority of patients with multiple liver cysts are asymptomatic, recognized only after routine ultrasound investigation. However, the most common clinical patterns include abdominal pain, dyspnea and early satiety related to a compartmental syndrome. In the present study, we described a rare association of PCLD with intracranial meningiomas in patients included on a liver transplant waiting list, focusing on the diagnosis, possible association with any chromosomal alteration and mandatory treatment of those lesions prior to transplantation.

\section{Case reports}

Two female patients, 39 and 49 years of age, were referred for evaluation for extensive abdominal tumors, with symptoms related to a compartmental syndrome, which included dyspnea, abdominal pain and early satiety. The two patients provided informed consent, and the study was approved by the ethics committee of Sao Paulo State University.

Clinical investigation included an abdominal ultrasound and further computer tomography (CT), which revealed an extensive liver involvement by cyst lesions, without kidney involvement (Fig. 1). The mean cyst diameters of the largest liver cysts were $10 \pm 5 \mathrm{~cm}$, located deeply in the liver parenchyma and the small ones, superficially.

Abnormal physical findings were similar in the two patients, and included right upper quadrant pain and abdominal distension.

Laboratorial data were collected and included creatinine, urea nitrogen, aspartate aminotransferase (AST), alanine aminotransferase (ALT), alkaline phosphatase (ALP), $\gamma$-glutamyl transferase (GGT), total bilirubin, RNI and prothrombin time, which were relatively stable and without significant abnormalities for the two patients. The same blood type, AB, was identified.

The main reasons for including the two patients on a liver transplant waiting list were the great liver size, which reached the lower abdominal quadrant, with diaphragmatic and inferior vena cava compression, leading to edema of the legs.

Screening for extrahepatic manifestation was performed using magnetic resonance imaging (MRI), which revealed the presence of a right frontal meningioma in the first patient 


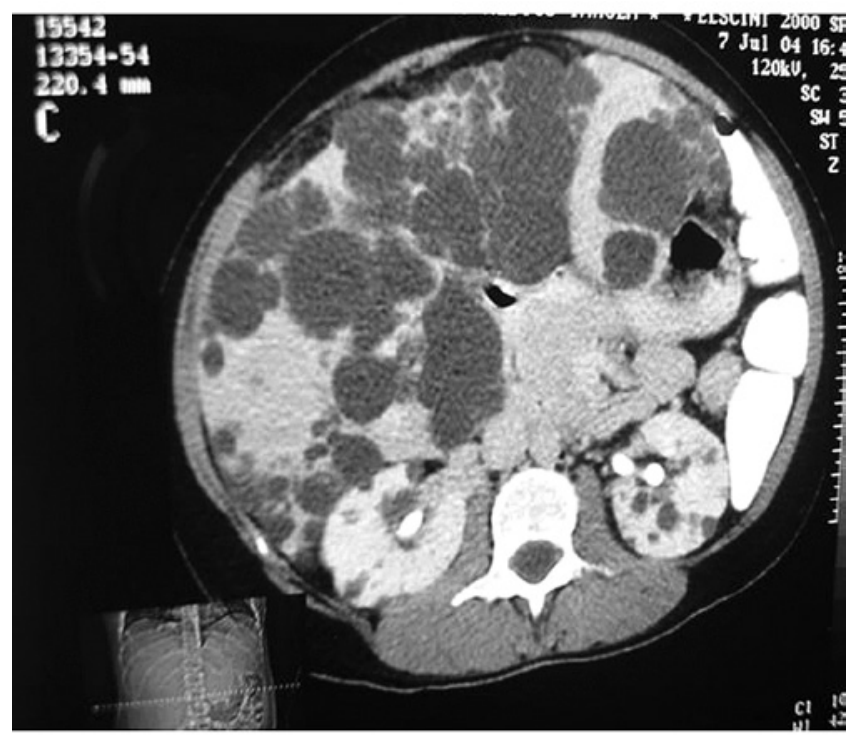

Figure 1. CT scan with an extensive polycystic liver disease. CT, computer tomography.

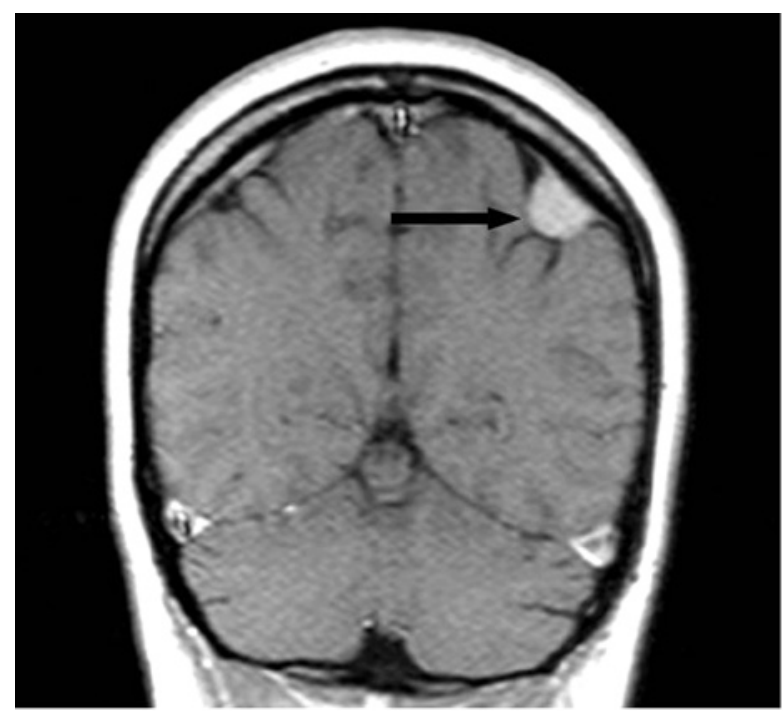

Figure 2. MRI with a right frontal meningioma. MRI, magnetic resonance imaging.

(Fig. 2), and a parietal posterior calcified meningioma, plus the presence of osteoma and encephalic calcification, in the second patient (Fig. 3), each measuring 1 and $7 \times 3 \times 2 \mathrm{~cm}$ in diameter, respectively. The patients were submitted to a craniotomy for tumor removal, with histological patterns compatible with fibrous meningioma in the first patient, and transitional meningioma in the second patient, both with psammomatous bodies (Fig. 4). These histological features lead to a classification of Grade I according to World Health Organization (WHO) criteria. The postoperative course was uneventful, with the patients still awaiting a liver transplant.

Post-operative conventional cytogenetic studies. Cells were obtained from the two patients and cultivated for 24 or $48 \mathrm{~h}$ in MarrowMAX ${ }^{\mathrm{TM}}$ Bone Marrow Medium (Gibco; Invitrogen,

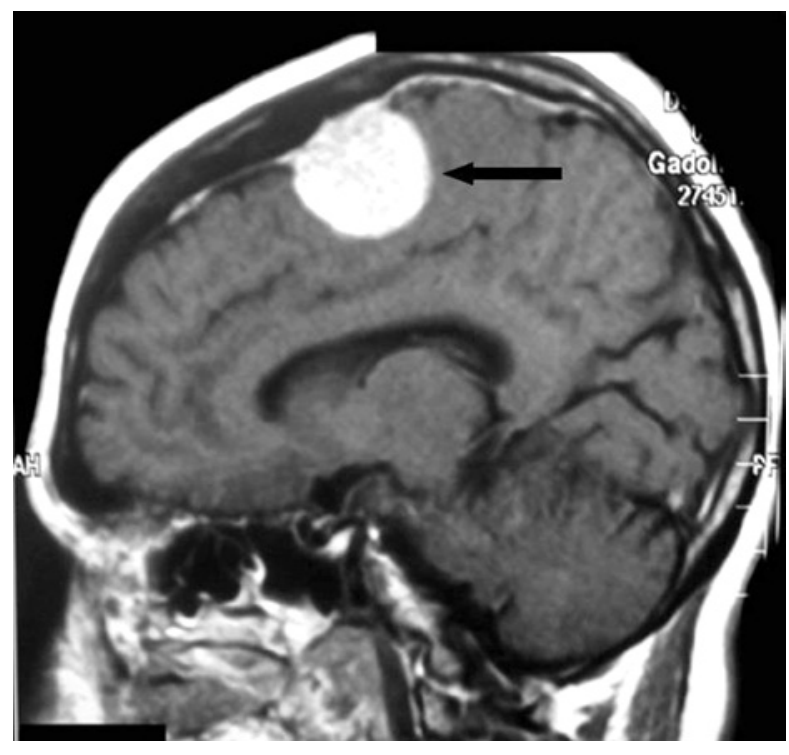

Figure 3. MRI with a parietal posterior calcified meningioma plus the presence of an osteoma and encephalic calcification. MRI, magnetic resonance imaging.

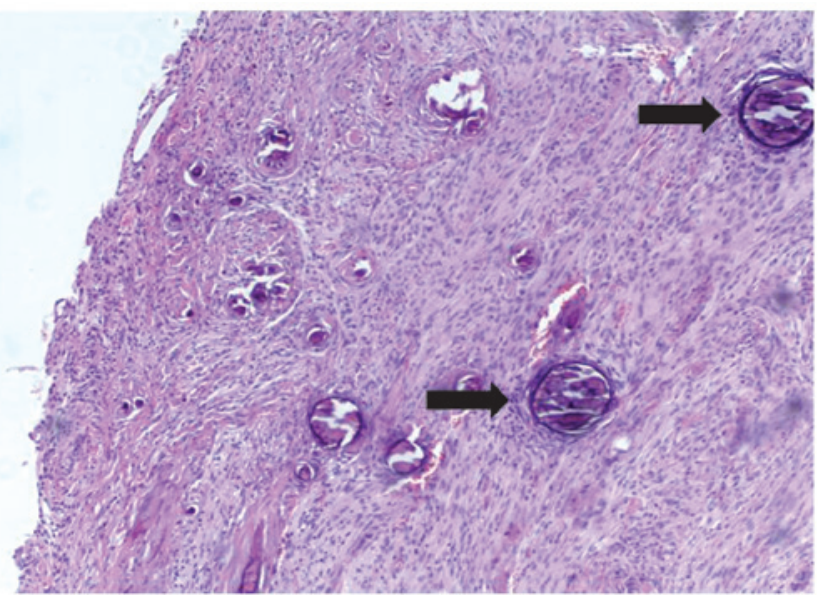

Figure 4. Meningioma. Black arrow, psammomatosous bodies.

Brazil) with $10 \%$ fetal calf serum without stimulation. The cells were then harvested using conventional techniques. The GTG banding analysis was performed according to Scheres (6). Metaphase chromosomes were analyzed using an Olympus BX61 microscope connected to BandView 4.5 software (Applied Spectral Imaging, Israel). Chromosomes were identified and classified according to the International System of Human Cytogenetic Nomenclature guidelines (7). The analysis of twenty metaphases for each patient did not reveal any cytogenetic changes.

\section{Discussion}

The symptoms of isolated PCLD are specifically correlated with the extension of liver involvement (8), with the possibility of life-threatening complications and a poor quality of life.

No exact epidemiological data are currently available regarding the incidence of PCLD in any population. However, 
PCLD, without ADPKD, is a rare condition with a prevalence of 0.05 or $0.13 \%$ in two autopsy series.

When associated with ADPKD, the prevalence reported was $16 \%$ in one study and $93 \%$ in another $(9,10)$. In these cases, a relevant genetic defect was linked to the polycystic kidney disease 1 (PKD1) locus on chromosome 16 (the main locus responsible for the disease) (12) and PKD2 locus on chromosome 4 . No evidence of further locus heterogeneity was described (11).

In a study conducted by Pirson et al (12) of a family with PCLD not associated with kidney cysts and transmitted through three generations, linkage to mutations of the genetic markers of PKD1 and PKD2 were excluded. One report concluded that isolated PCLD exists as a genetic disease, distinct from ADPKD1 and ADPKD2.

A third gene, protein kinase $\mathrm{C}$ substrate $80 \mathrm{~K}-\mathrm{H}$ (PRKCSH), accounts for a comparatively rare, isolated form of autosomal dominant PCLD, which displays no kidney involvement (4). Waanders et al (13) concluded that $16 \%$ of patients with PCLD were found to have either PRKCSH or SEC63 mutations.

In a study of 20 individuals with PCLD, Yang et al (14) reported that the PRKCSH gene was not a major genetic cause, and that there may be at least another locus responsible for the disease in Taiwanese patients. Through analysis of eight Finnish families with PCLD, Tahvanainen et al (15) concluded that, in most families, PCLD is linked to a locus on chromosome 19p13.2-13.1; however, the disease is genetically heterogeneous with at least one more locus, which remains to be found.

Associated morbidities were notably less frequent in patients with PCLD when comparing individuals with PCLD linked to ADPKD16, and included mitral valve prolapse, diverticuli, brain aneurisms and intracranial arachnoid cysts (17).

Intracranial lesions in association with polycystic diseases are typically incidental findings in routine investigations, while symptomatic lesions may include headaches, seizure or focal neurological deficits (18). However, the association of rare, developmental, solid tumors in meningeal membrane (meningiomas) with PCLD has yet to be reported.

The treatment of PCLD aims to symptomatically relieve symptoms related to cysts, and includes conservative approaches such as cyst decompression, liver resections or liver transplantation. The main reason for the consideration of liver transplantation in our patients was the large liver volumes, leading to an abdominal compartmental syndrome.

The meningeal tumors were treated prior to liver transplantation for obvious reasons, since the tumor measurement following resection was $7 \times 3 \times 2 \mathrm{~cm}$ in our second patient.

Meningiomas are mostly benign tumors, classified as Grade I, originating from the arachnoid cap cells and represent $13-26 \%$ of all intracranial tumors (19). They are more common in older age individuals and in females. The role of hormones has yet to beclarified, with a five-year survival of more than $80 \%$ in typical meningiomas, but is poorer in atypical presentations. Papillary and haemangiopericytic morphology, large tumors, high mitotic index, absence of progesterone receptors, deletion and loss of heterozygosis are all poor prognostic factors. Complete surgical excision is the standard treatment. Radiotherapy may be used in the clinical practice in atypical Grade II and malignant Grade III or recurrent meningiomas (20).
The histological characterization of fibrous and transitional meningiomas in the patients we studied lead to a classification of Grade I according to WHO criteria (19).

The postoperative follow-up for the two patients was uneventful, without complications, with the patients remaining on the liver transplant waiting list.

Bearing in mind that the polymorphism is a feature of extrahepatic lesions in PCLD, and is either associated, or not, to ADPKD, improved awareness is mandatory in order to determine the best approach for the treatment of these patients. The lack of a genetic or familial association between these two cases shows they are likely to have occurred by chance rather than represent a previously unrecognized association between polycystic liver disease and cranial meningioma.

The analysis of a large patient cohort may provide us with more detailed information and a better understanding of the genetic background of such occurrences.

At present, a more prudent course toward the identification of PCLD remains a combination of refinement of the genetic interval and continued gene identification based on the genomic sequence of the region, with emphasis placed on the understanding of liver cyst pathogenesis, which may lead to non-surgical therapy for patients affected with symptomatic liver disease (21).

\section{Acknowledgements}

All authors contributed to the study design and approved the final version of the manuscript.

\section{References}

1. Schivink WI, Huston J, Torres VE and Marsh WR: Intracranial cysts in autosomal dominant polycystic kidney disease. J Neurosurg 83: 1004-1007, 1995.

2. Romao EA, Moyses Neto M, Teixeira SR, Muglia VF, Vieira OM and Dantas M: Renal and extrarenal manifestations of autosomal dominant polycystic kidney disease. Braz J Med Biol Res 39: 533-538, 2006.

3. Masyuk T and LaRusso N: Polycystic liver disease: new insights into disease pathogenesis. Hepatology 43: 906-908, 2006.

4. Everson GT, Taylor MRG and Doctor RB: Polycystic disease of the liver. Hepatology 40: 774-782, 2004.

5. Portmann BC and Roberts EA: Developmental abnormalities and liver disease in childhood. In: MacSweens Pathology of the Liver. Burt AD, Portmann BC, Ferrell LD (eds). Churchill Livingstone Elsevier, China, pp174-176, 2007.

6. Scheres JM: Identification of two robertsonian translocations with a giemsa banding technique. Hum Genet 15: 253-256, 1972.

7. Mitelman F (ed): ISCN: An International System for Human Cytogenetic Nomenclature. Karger, Basel, p7, 2005.

8. Shrestha R, Martina JA, Kerkhof R, et al: Postmenopausal estrogen therapy selectively stimulates hepatic enlargement in women with autosomal dominant polycystic kidney disease. Hepatology 26: 1282-1286, 1997.

9. Karhunen PJ and Tenhu M: Adult polycystic liver and kidney diseases are separate entities. Clin Genet 30: 29-37, 1986.

10. Kwok MK and Lewin KJ: Massive hepatomegaly in adult polycystic liver disease. Am J Surg Pathol 12: 321-324, 1988.

11. Peters DJM, Spruit L, Saris JJ, Ravine D, Sandkuijl LA, Fossdal R, Boersma J, et al: Chromosome 4 localization of a second gene for autosomal dominant polycystic kidney disease. Nat Genet 5: 359-362, 1993.

12. Pirson Y, Lannoy N, Peters D, Geubel A, Gogot JF, Breuning M and Dumoulin CV: Isolated polycystic liver disease as a distinct genetic disease, unlinked to polycystic kidney disease 1 and polycystic disease 2. Hepatology 23: 249-252, 1996. 
13. Waanders E, Morsche RH, Man RA, Jansen JB and Drenth JP: Extensive mutational analysis of PRKCSH and SEC63 broadens the spectrum of polycystic liver disease. Hum Mutat 27: 830-836, 2006.

14. Yang AM, Shi SC, Chu CH, Wang TE and Yang WS: PRKCSH genetic mutation was not found in taiwanese patients with polycystic liver disease. Dig Dis Sci 24: 1-15, 2009.

15. Tahvanainen $\mathrm{P}$, Tahvanainen E, Reijonen H, Halme L, Kaariainen $\mathrm{H}$ and Hockerstedt $\mathrm{K}$ : Polycystic liver disease is genetically heterogeneous: clinical and linkage studies in eight Finnish families. J Hepatology 38: 39-43, 2003.

16. Hoevenaren IA, Wester R, Schrier RW, MacFann K, Doctor RB, Drenth JP and Everson GT: Polycystic liver: clinical characteristics of patients with isolated polycystic liver disease compared with patients with polycystic liver and autosomal dominant polycystic kidney disease. Liver Int 28: 264-270, 2008.

17. Leung GKT and Fan YW: Chronic subdural haematoma and arachnoid cyst in autosomal dominant polycystic kidney disease (ADPKD). J Clin Neurosci 12: 817-819, 2005.
18. Galassi E, Tognetti F, Gaist G, Fagioli L and Frank G: CT scan and metrizamide CT cisternography in arachnoid cysts of the middle cranial fossa: classification and pathophysiological aspects. Surg neurol 17: 363-369, 1982.

19. Louis DN, Scheithauer BW, Budka H, Van Deimling A and Kepes JJ: Meningiomas. In: Pathology and Genetics Tumours of the Nervous System, WHO Classification of Tumours. Kleihues P and Cavenee WK (eds). Iaro press, Lyon, pp176-184, 2000.

20. Marosi C, Hassler M, Roessler K, et al: Meningioma. Crit Rev Oncol Hematol 67: 153-171, 2008.

21. Reynolds DM, Falk CT, Li A, King BF, Kamath PS, Huston J, et al: Identification of a locus for autosomal dominant polycystic liver disease, on chromosome 19p13.2-13.1. Am J Hum Genet 67: 1598-1604, 2000. 